\title{
Erratum to: Revisited: Borrelia burgdorferi sensu lato infections in hard ticks (Ixodes ricinus) in the city of Hanover (Germany)
}

Julia Tappe ${ }^{1}$, Daniela Jordan ${ }^{1}$, Elisabeth Janecek ${ }^{1}$ Volker Fingerle ${ }^{2}$ and Christina Strube ${ }^{1^{*}}$

\section{Erratum}

Unfortunately, we noticed that in our published paper [1] the numbers of ticks co-infected with Borrelia burgdorferi (sensu lato) (s.l.) and Anaplasma phagocytophilum were partly incorrect as one adult female tick was detected false-positive for A. phagocytophilum infection. Corrected data showed that none of the female or adult ticks was co-infected with Borrelia burgdorferi (s.l.) and Anaplasma phagocytophilum. Among all tick stages, only six instead of seven out of 2,100 ticks were infected with both pathogens, but the prevalence of $0.3 \%$ reported in [1] remained unchanged. The corrected Table 4 is included below. The authors would like to apologise for any inconvenience caused.

\footnotetext{
* Correspondence: christina.strube@tiho-hannover.de

${ }^{1}$ Institute for Parasitology, University of Veterinary Medicine, Hanover,

Buenteweg 17, Hannover 30559, Germany

Full list of author information is available at the end of the article
} 
Table 4 Co-infections with B. burgdorferi (s.l.) and Rickettsiales in Hanoverian ticks in 2010

\begin{tabular}{|c|c|c|c|c|c|c|}
\hline & $\begin{array}{l}\text { No. of collected } \\
\text { ticks }\end{array}$ & $\begin{array}{l}\text { No. of } B \text {. burgdorferi } \\
\text { (s.l.) positive ticks }\end{array}$ & Total co-infections & $\begin{array}{l}\text { Rickettsia spp. } \\
\text { co-infections }\end{array}$ & $\begin{array}{l}\text { A. phagocytophilum } \\
\text { co-infections }\end{array}$ & $\begin{array}{l}\text { Co-infections with } \\
\text { Rickettsia spp. and } \\
\text { A. phagocytophilum }\end{array}$ \\
\hline & & №. (\%) & No. (\%) & No. (\%) & No. (\%) & No. (\%) \\
\hline Adults & 372 & $124(33.3)$ & 43 (11.6) & $42(11.3)$ & $0\left(n a^{*}\right)$ & $0\left(n a^{*}\right)$ \\
\hline Males & 196 & $58(29.6)$ & $22(11.2)$ & $22(11.2)$ & $0\left(n a^{*}\right)$ & $0\left(n a^{*}\right)$ \\
\hline Females & 176 & $66(37.5)$ & $21(11.9)$ & $20(11.4)$ & $0\left(n a^{*}\right)$ & $0\left(n a^{*}\right)$ \\
\hline Nymphs & 1697 & $344(20.3)$ & $120(7.1)$ & $111(6.5)$ & $6(0.4)$ & $3(0.2)$ \\
\hline Larvae & 31 & $8(25.8)$ & $0\left(n a^{*}\right)$ & $0\left(n a^{*}\right)$ & $0\left(n a^{*}\right)$ & $0\left(n a^{*}\right)$ \\
\hline All stages & 2,100 & $476(22.7)$ & $163(7.8)$ & $153(7.3)$ & $6(0.3)$ & $3(0.1)$ \\
\hline
\end{tabular}

*na, not applicable

\section{Author details}

'Institute for Parasitology, University of Veterinary Medicine, Hanover,

Buenteweg 17, Hannover 30559, Germany. ${ }^{2}$ German National Reference

Centre for Borrelia, Veterinärstraße 2, Oberschleißheim 85764, Germany.

Received: 18 April 2016 Accepted: 18 April 2016

Published online: 29 April 2016

\section{Reference}

1. Tappe J, Jordan D, Janecek E, Fingerle V, Strube C. Revisited: Borrelia burgdorferi sensu lato infections in hard ticks (Ixodes ricinus) in the city of Hanover (Germany). Parasit Vectors. 2014;7:441. doi:10.1186/1756-3305-7-441.

Submit your next manuscript to BioMed Central and we will help you at every step:

- We accept pre-submission inquiries

- Our selector tool helps you to find the most relevant journal

- We provide round the clock customer support

- Convenient online submission

- Thorough peer review

- Inclusion in PubMed and all major indexing services

- Maximum visibility for your research

Submit your manuscript at www.biomedcentral.com/submit
C) Biomed Central 